\title{
Per una scuola che guarda all'Europa e al plurilinguismo
}

\section{Elena Maria Grosso}

\section{(2) OpenEdition \\ 1 Journals}

Edizione digitale

URL: http://journals.openedition.org/esp/1080

DOI: $10.4000 /$ esp.1080

ISSN: 2532-0319

Editore

Centre d'Information sur l'Éducation Bilingue et Plurilingue

\section{Edizione cartacea}

Data di pubblicazione: 1 giugno 2017

Paginazione: 19-25

ISSN: 1127-266X

Notizia bibliografica digitale

Elena Maria Grosso, «Per una scuola che guarda all'Europa e al plurilinguismo », Éducation et sociétés plurilingues [Online], 42 | 2017, Messo online il 01 février 2018, consultato il 15 septembre 2020. URL : http://journals.openedition.org/esp/1080 


\section{PER UNA SCUOLA CHE GUARDA ALL'EUROPA E AL PLURILINGUISMO}

\section{Elena Maria Grosso}

En Vallée d'Aoste l'école est de plus en plus ouverte au bi-plurilinguisme et à l'emploi de pratiques didactiques innovantes. L'évolution de son modèle d'éducation bi-plurilingue découle d'une série de normes régionales qui, en application de son Statut d'autonomie, ont permis d'adapter le système scolaire national ainsi que les curriculums de tous les niveaux scolaires aux nécessités locales, notamment pour ce qui concerne l'enseignement des langues. L'article présente les traits essentiels de cette réforme.

Mots-clés: réforme scolaire, approches plurielles, éducation bilingue, plurilinguisme, didactique expérimentale.

In the Aosta Valley, schools are more and more open to bi- and multilingualism and to the use of groundbreaking didactic methods. The evolution of its bi-multilingual educational model is the outcome of a series of norms which, thanks to the implementation of its status as an autonomous region, allowed adapting the national education system as well as the curricula at all levels to local needs, particularly as concerns language teaching. The article presents the main features of this reform.

Key-words: school reform, pluralistic approaches, bilingual education, multilingualism, experimental didactics

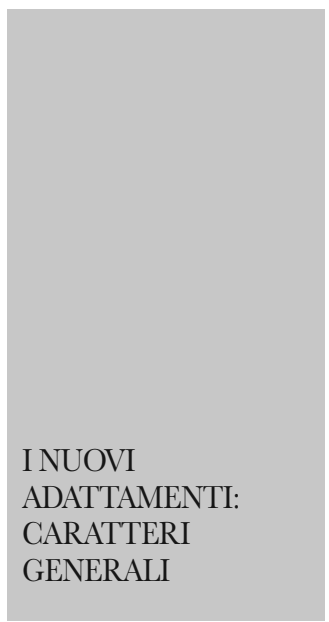

Tn Valle d'Aosta la scuola si apre sempre più al bi-plurilinguismo e all'utilizzo di pratiche didattiche innovative. Questo grazie ad un Protocollo d'intesa siglato nel luglio 2015 tra la Regione e il Ministero dell'Istruzione, dell'Università e della Ricerca italiano, all'approvazione - da parte del governo regionale - di un documento che adatta le Indicazioni nazionali per il curricolo alle necessità locali, in particolare modo per quanto riguarda gli insegnamenti linguistici, alla legge regionale 3 agosto 2016 n.18 che prevede disposizioni per l'armonizzazione della legge nazionale del 13 luglio 2015 n. 107, la così detta "Buona scuola".

Lo sviluppo di una educazione bi-plurilingue aperta all'Europa e il rispetto delle differenze culturali e linguistiche già promossi, oltre che dallo Statuto (1) della Valle d'Aosta, da diverse leggi regionali riguardanti sia l'attuazione dell'autonomia scolastica che disposizioni in materia di organizzazione del personale, a 


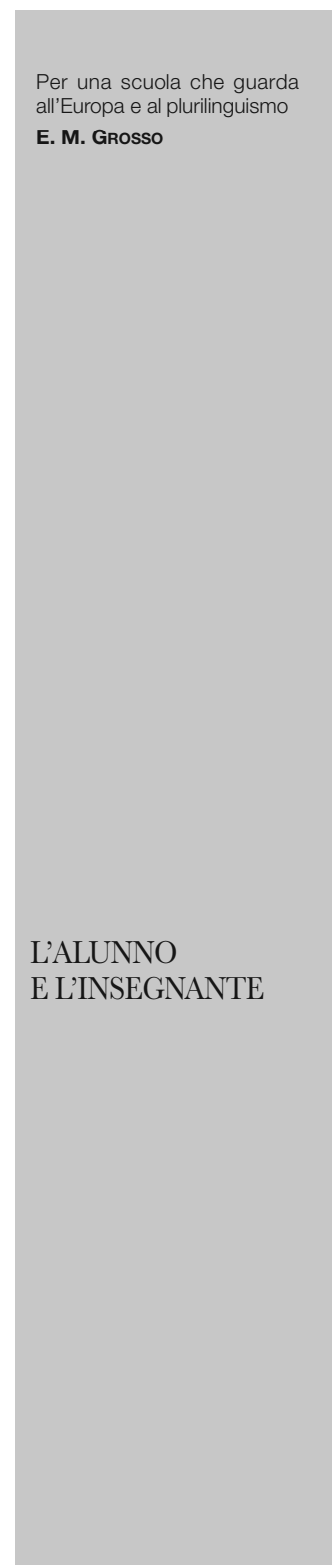

partire dal corrente anno scolastico, riceveranno un nuovo impulso.

Questo grazie all'avvio della sperimentazione, in tutte le classi prime, degli "Adattamenti" alle necessità locali della Valle d'Aosta delle Indicazioni nazionali per il curricolo della scuola dell'infanzia, del primo ciclo di istruzione e dei piani di studio del secondo ciclo di istruzione (2), adattamenti già preconizzati dal Protocollo di Intesa tra il Ministero dell'Istruzione, dell'Università e della Ricerca e la Regione autonoma Valle d'Aosta del 25 luglio 2015.

L'obiettivo è chiaramente quello di riconoscere, rinforzare e valorizzare le competenze bi-plurilingui degli alunni valdostani. Competenze che aprano loro prospettive di successo personale e professionale, da lungo promosse dal Consiglio d'Europa, parti essenziali della nuova strategia "Europa 2020" e del Piano di interventi per il miglioramento e il potenziamento delle politiche educative previsto a livello regionale nel maggio 2014.

Gli attuali Adattamenti, tenendo conto dell'evoluzione normativa avvenuta dagli anni ottanta ad oggi, vogliono infatti avvicinarsi al nuovo contesto europeo e valorizzare le buone pratiche linguistiche e didattiche messe in atto nelle scuole della Regione in tanti anni. Colmano inoltre quel vuoto lasciato per lungo tempo nella scuola secondaria di secondo grado per la quale gli Adattamenti sino ad oggi - sono mancati (3).

Essi disegnano il profilo di un alunno che si trova ad essere parte integrante di un sistema in cui più lingue sono insegnate e a cui vengono richiesti atteggiamenti di apertura, di curiosità e di rispetto nei confronti della diversità. E' un alunno in grado di utilizzare le conoscenze e le competenze di cui dispone in una lingua per comprendere e produrre in un'altra lingua, che si sente a suo agio in situazioni di comunicazione in cui più lingue e o culture sono presenti, che affronta compiti il più possibile autentici e che favoriscono lo sviluppo di una dimensione collaborativa e di una interazione orale. E' un alunno che apprende ad utilizzare più lingue e che utilizza più lingue per apprendere, cosciente del fatto che acquisizione del contenuto e acquisizione linguistica vanno di pari passo.

In tale contesto anche l'insegnante assume un nuovo ruolo: oltre ad essere un tutore che aiuta gli studenti nella costruzione della percezione del proprio sé, del processo di apprendimento e di 


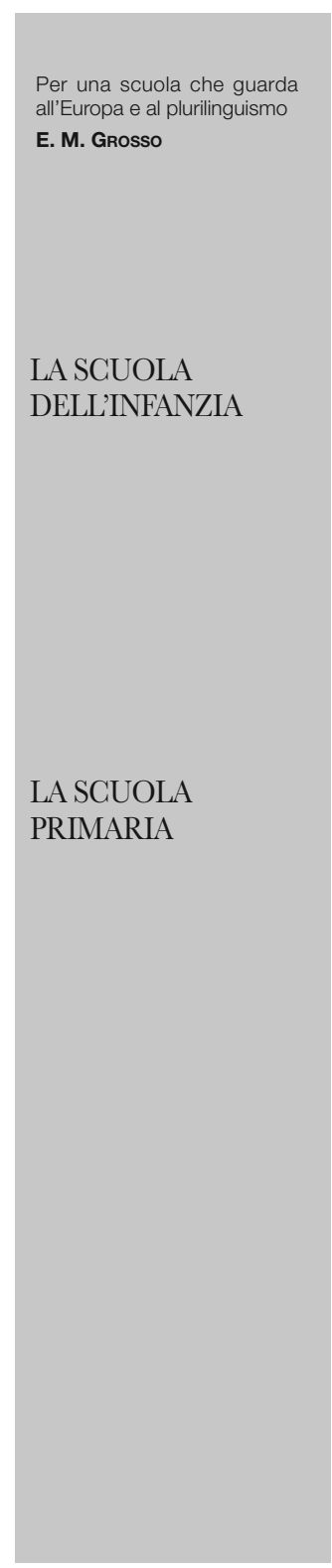

autovalutazione del proprio operato, diventa un facilitatore dell'apprendimento che mette in atto strategie di problem solving e di cooperative learning. Un insegnante che, attento ai molteplici stili di apprendimento e alle diversità, segue passo dopo passo l'evoluzione degli apprendimenti linguistici, li valorizza e ne tiene traccia grazie all'utilizzo di strumenti validati a livello europeo quali il Portfolio Europeo delle Lingue, il Passaporto Europass, il Quadro Comune europeo di riferimento per le lingue e quello per gli approcci plurali.

Per la scuola dell'infanzia, come è previsto dall'art. 39 dello Statuto, gli Adattamenti confermano l'insegnamento per tempi uguali della lingua italiana e di quella francese in tutte le attività didattiche. Diverse le modalità di organizzazione che possono integrarsi, a seconda delle esigenze del contesto e delle risorse disponibili: curriculum bilingue e sviluppo delle competenze, un docente/una lingua, mezza giornata in italiano/mezza giornata in francese. Vengono introdotte inoltre azioni di "éveil aux langues", cioè attività di scoperta della diversità linguistica e culturale tramite un approccio ludico alla lingua inglese, a quella tedesca e alle lingue familiari al bambino quali il francoprovenzale.

Nella scuola primaria la conferma della parità oraria dell'insegnamento della lingua italiana e di quella francese è accompagnata da una più precisa formalizzazione dell'utilizzo delle lingue nell'insegnamento delle diverse discipline.

Se in francese sono approfonditi gli argomenti di interesse locale di storia e geografia, un'educazione ed alcuni obiettivi di matematica e scienze, la lingua inglese (o tedesca nella Comunità Walser) - insegnata per due ore settimanali - viene utilizzata almeno una terza ora per l'insegnamento di discipline non linguistiche, più precisamente per la parte sperimentale delle scienze e per un'educazione a scelta dell'Istituzione scolastica, con preferenza per educazione motoria.

$\mathrm{Al}$ termine dei cinque anni di corso, l'obiettivo per le lingue straniere è il raggiungimento di una competenza linguistica Al del Quadro Comune di riferimento per le lingue (QCER).

In tale contesto evidenti il ruolo fondamentale assunto dalla progettazione collegiale del curriculo plurilingue al fine di prevenire la frammentazione dell'insegnamento, l'utilizzo di un approccio laboratoriale fondato su esperienze graduali e concrete che può prevedere l'uso di fonti autentiche tratte dal web e materiali audio 


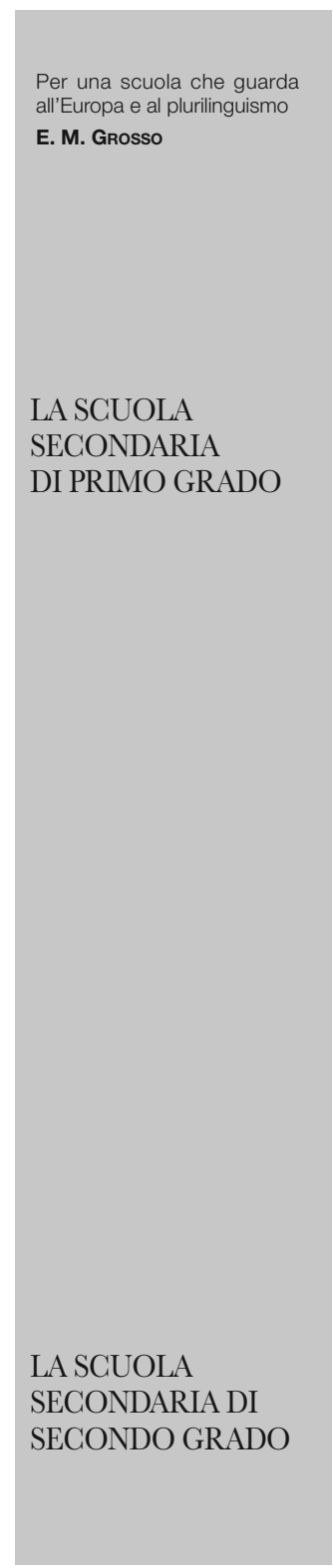

predisposti appositamente per le attività didattiche.

Anche per la scuola secondaria di primo grado i nuovi Adattamenti, a differenza di quelli del 1994, definiscono quali discipline svolgere nelle diverse lingue. Sono insegnate in lingua francese la geografia, la musica e parte della storia; in lingua inglese le scienze, l'educazione fisica e parte di tecnologia (informatica). Per la Comunità Walser l'individuazione delle discipline da insegnare in lingua tedesca è definita nel progetto d'istituto.

Sempre il progetto d'istituto è tenuto ad indicare il monte ore annuale da destinare all'insegnamento in lingua francese o in lingua straniera (inglese/tedesco) di storia, tecnologia, arte e religione consacrando almeno uno spazio del 30\% del monte ore di queste singole discipline all'attività svolta in lingua.

L'insegnamento delle discipline non linguistiche in lingua viene tuttavia introdotto in modo graduale al fine di permettere adeguata formazione del corpo docenti; l'obiettivo da raggiungere, al termine del primo triennio di attuazione degli Adattamenti, è che il $50 \%$ del monte ore delle discipline non linguistiche sia veicolato in lingua francese e il $30 \%$ in lingua straniera (inglese o tede$\mathrm{sco}$ ).

Per favorire la condivisione e l'ampliamento di percorsi integrati di lingua e disciplina, si prevedono forme modulari di apprendimento, attività laboratoriali, classi aperte, realizzazione di progetti interdisciplinari, utilizzo di tecnologie multimediali, scambi tra scuole sia in presenza sia a distanza tramite videoconferenze, webinar e eTwinning.

Le competenze bi-plurilingui acquisite nel percorso di studio trovano infine adeguata collocazione nel modello di Certificazione delle competenze al termine del primo ciclo di istruzione redatto a livello regionale e che richiama le otto Competenze chiave per l'apprendimento permanente proposte dal Parlamento europeo nel 2006.

Innovazioni pure per la scuola secondaria di secondo grado, mai approdata, prima d'ora, agli Adattamenti previsti dall'art. 40 dello Statuto della Valle d'Aosta.

Nonostante la realizzazione, a partire da metà de gli anni 90, di diverse attività interdisciplinari e di numerosi progetti educativi e 


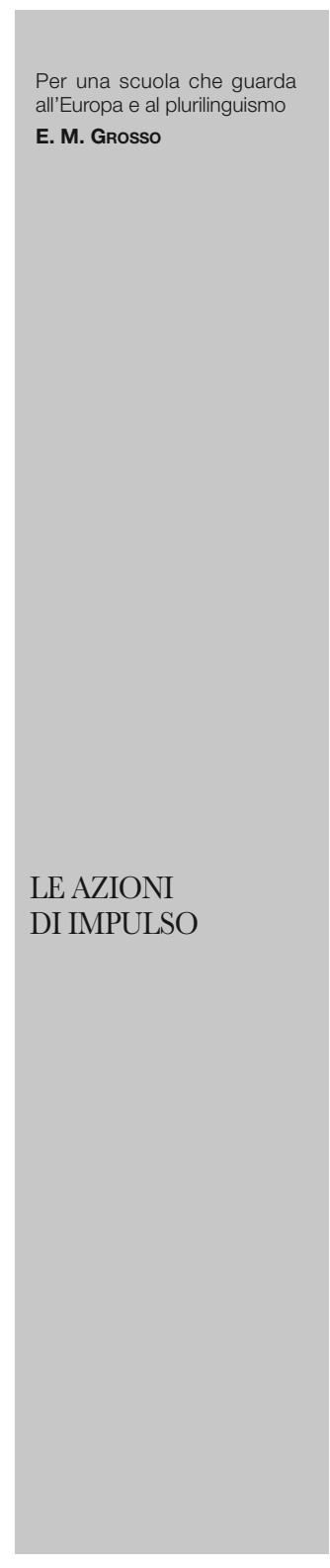

didattici bi-plurilingui quali Classes Villette, Classes internationales, Classes Musées, nelle scuole superiori, l'istituto del francese - ad esclusione di alcuni percorsi - ha in genere goduto per tutti questi anni di semplice parità oraria con l'italiano.

A partire da quest'anno scolastico la deliberazione degli Adattamenti determina, sempre nelle classi prime di tutte le scuole secondarie di secondo grado, l'insegnamento in francese di discipline non linguistiche dell'area storico - sociale. Ogni collegio docenti è tenuto ad individuare le discipline più appropriate in base al curriculum della scuola e alle risorse disponibili.

È così che nelle classi prime dei licei e degli istituti tecnici, vengono garantiti almeno 66 moduli orari annui di insegnamento integrato di lingua francese e disciplina; negli istituti professionali almeno 33 .

Si suggerisce sempre l'applicazione di una didattica laboratoriale che favorisca l'esplorazione, la ricerca, che incoraggi un apprendimento collaborativo e che valorizzi il "saper fare" di ogni alunno.

Per l'introduzione della lingua inglese in discipline non linguistiche dell'area tecnica-scientifica, bisognerà attendere il 2019/20, in una logica di continuità con la sperimentazione attuata da quest'anno nella scuola secondaria di primo grado.

Per una adeguata applicazione degli Adattamenti, si prevedono azioni di accompagnamento e di costante monitoraggio al fine di predisporre eventuali correttivi.

È così che per potenziare le competenze linguistiche e metodologiche degli insegnanti di tutti i gradi di scuola continuerà, almeno per un triennio, l'attività di formazione già messa in atto lo scorso anno. Le formazioni riguarderanno sia l'ambito del francese nonostante i docenti siano in genere già in possesso della certificazione di piena conoscenza di tale lingua -, sia quello dell'inglese. Per la lingua inglese, che diventa elemento significativo all'interno del sistema educativo, l'obiettivo è quello di fare acquisire ai docenti dell'infanzia un livello di competenza linguistica B1, a quelli di scuola primaria un livello B2, a quelli di discipline non linguistiche di scuola secondaria di primo e secondo grado che insegnano la loro disciplina in inglese un livello C1 (QCER). Le attività di formazione potranno essere rinforzate grazie alla predisposizione di materiali didattici bi-plurilingui, all'accoglienza di docenti francofoni e anglofoni nelle scuole e da eventuali interventi di esperti esterni. 


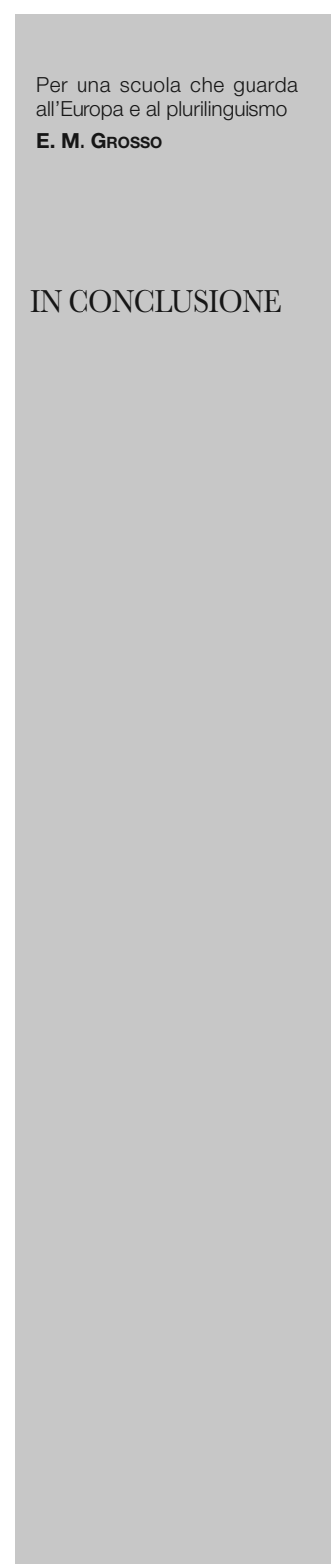

Altre azioni di accompagnamento mirano invece a riflettere sulla continuità dell'insegnamento bi - plurilingue dalla scuola dell'infanzia alla secondaria di secondo grado al fine di garantire una coerenza tra i diversi cicli e a facilitare la progressione delle competenze degli alunni, a sostenere le istituzioni scolastiche affinché tutte lavorino per il conseguimento di competenze bi- plurilingui chiare e condivise.

L'anno scolastico 2016-2017 vedrà pertanto la costituzione di diversi gruppi di lavoro con docenti delle differenti istituzioni scolastiche, coordinati da dirigenti e da dirigenti tecnici, con il compito di definire il profilo dell'alunno in uscita dai diversi gradi di scuola per quanto concerne le competenze linguistiche e plurilingui, i curricoli verticali delle aree storica e scientifica in contesto bi-plurilingue, gli strumenti di osservazione e i traguardi di competenza.

Prevista anche l'istituzione di un gruppo di lavoro per la definizione delle prove standardizzate di francese, inglese e tedesco previste dal Decreto Legislativo 3 marzo 2016 n. 44/2016 e che, sempre a partire da quest'anno scolastico, si affiancano alle prove Invalsi. Si rivela dunque ancora più chiaramente necessaria la definizione dei livelli di competenza linguistica richiesta nei vari step del percorso scolastico e la rilevazione di dati affidabili sulle acquisizioni linguistiche degli alunni al fine di attuare una riflessione sugli stessi e predisporre eventuali azioni di miglioramento.

La possibilità inoltre di calibrare gli esami finali che verificano il livello di padronanza linguistica sui livelli di competenza definiti dal QCER sarebbe un'occasione per un riconoscimento internazionale della competenza acquisita. 


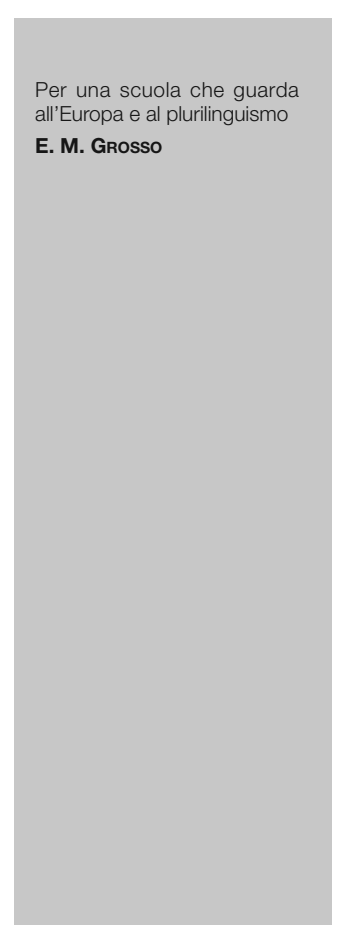

È evidente che gli Adattamenti, affiancati dalla nuova legge regionale di riforma (4), aprono un nuovo percorso che vuole innalzare la qualità e l'efficacia del sistema educativo valdostano, favorire la mobilità e l'occupabilità dei giovani e che vuole operare - secondo la strategia europea - per una crescita "intelligente, sostenibile e inclusiva". Sarà la partecipazione di tutti gli attori del sistema a determinarne la riuscita, a cogliere l'occasione per offrire alle nuove generazioni un'opportunità di ulteriore arricchimento che potrà facilitare loro l'ingresso in un mondo del lavoro caratterizzato da dinamismo, spazi di ascolto, collaborazione e flessibilità.

NOTE

(1) Legge costituzionale n.4 del 26 febbraio 1948. Artt. 39-40: in Valle d'Aosta vengono riconosciute due lingue ufficiali (italiano/francese) e gli adattamenti dell'insegnamento alle necessità locali. Art 40 bis: viene garantito l'insegnamento della lingua tedesca nei comuni della Valle del Lys.

(2) D.G.R. 19 agosto 2016, n.1103 (Adattamenti alle necessità locali della Valle d'Aosta delle Indicazioni nazionali per il curricolo della scuola dell'infanzia e del primo ciclo di istruzione e delle Indicazioni nazionali - piani di studio - delle scuole del secondo ciclo di istruzione).

(3) Legge regionale 27 dicembre 1996, n.50 (Interventi propedeutici all'applicazione degli articoli 39 e 40 dello Statuto speciale della Valle d'Aosta, approvato con legge costituzionale 26 febbraio 1948 n.4, nelle scuole secondarie di secondo grado).

(4) Legge regionale 3 agosto 2016, n. 18 - Disposizioni per l'armonizzazione della legge 13 luglio 2015, n.107 (Riforma del sistema nazionale di istruzione e formazione e delega per il riordino delle disposizioni legislative vigenti), con l'ordinamento scolastico della Valle d'Aosta. 